\title{
Permutation Arrays Under the Chebyshev Distance * $^{*}$
}

\author{
Torleiv Kløve, ${ }^{\dagger}$ Te-Tsung Lin, Shi-Chun Tsai, and Wen-Guey Tzeng ‡
}

October 31, 2018

\begin{abstract}
An $(n, d)$ permutation array (PA) is a subset of $S_{n}$ with the property that the distance (under some metric) between any two permutations in the array is at least $d$. They became popular recently for communication over power lines. Motivated by an application to flash memories, in this paper the metric used is the Chebyshev metric. A number of different constructions are given as well as bounds on the size of such PA.
\end{abstract}

\section{Introduction}

Let $S_{n}$ denote the set of all permutations of length $n$. A permutation array of length $n$ is a subset of $S_{n}$. Recently, Jiang et. al [1, 2] showed an interesting new application of permutation arrays for flash memories, where they used different distance metrics to investigate efficient rewriting schemes. Under the multilevel flash memory model, we find the Chebyshev metric very appropriate for studying the recharging and error correcting issues. We note it by $d_{\max }$. For $\pi, \sigma \in S_{n}, d_{\max }(\pi, \sigma)=\max _{i}\left|\pi_{i}-\sigma_{i}\right|$. We consider a noisy channel where pulse amplitude modulation (PAM) is used with different amplitude levels for each permutation symbol. The noise in the channel is an independent Gaussian distribution with zero mean for each position. The received sequence is the original permutation distorted by Gaussian noise, and its ranking can be seen as a permutation, which can be different from the original one.

To study the correlations between ranks, several metrics on permutations were introduced, such as the Hamming distance, the minimum number of transpositions taking one permutation to another, etc. 3], 4]. For instance, Stoll

\footnotetext{
*The research was supported in part by the National Science Council of Taiwan under contracts NSC-95-2221-E-009-094-MY3, NSC-96-2221-E-009-026, NSC-96-3114-P-001-002-Y and NSC-96-2219-E-009-013 and by the Norwegian Research Council.

${ }^{\dagger} \mathrm{T}$. Kløve is with the Department of Informatics, University of Bergen, N-5020 Bergen, Norway (Email: Torleiv.Klove@ii.uib.no).

${ }_{\ddagger}$ T.-T. Lin, S.-C. Tsai and W.-G. Tzeng are with the Department of Computer Science, National Chiao Tung University, Hsinchu 30050, Taiwan (Email: atman.cs94g@nctu.edu.tw, sctsai@csie.nctu.edu.tw, wgtzeng@cs.nctu.edu.tw).
} 
and Kurz [5] investigated a detection scheme of permutation arrays using Spearman's rank correlation. Chadwick and Kurz [6] studied the permutation arrays based on Kendall's tau.

Under the model of additive white Gaussian noise (AWGN) [7, there is only a small probability for any amplitude level to deviate significantly from the original one. This inspired us to use the Chebyshev distance. Observe that two permutations with a large Hamming distance can actually have a small Chebyshev distance and vice versa. They appear to complement each other in some sense.

In this paper, we give a number of constructions of PAs. For some we give efficient decoding algorithms. We also consider encoding from vectors into permutations.

\section{Notations}

We use $[n]$ to denote the set $\{1, \ldots, n\} . S_{n}$ denotes the set of all permutations of $[n]$. For any set $X, X^{n}$ denotes the set of all $n$-tuples with elements from $X$.

Let $\iota$ denote the identity permutation in $S_{n}$. The Chebyshev distance between two permutations $\pi, \sigma \in S_{n}$ is

$$
d_{\max }(\pi, \sigma)=\max \left\{\left|\pi_{j}-\sigma_{j}\right| \mid 1 \leq j \leq n\right\} .
$$

An $(n, d)$ permutation array $(\mathrm{PA})$ is a subset of $S_{n}$ with the property that the Chebyshev distance between any two distinct permutations in the array is at least $d$. We sometimes refer to the elements of a PA as code words.

The maximal size of an $(n, d) \mathrm{PA}$ is denoted by $P(n, d)$. Let $V(n, d)$ denote the number of permutations in $S_{n}$ within Chebyshev distance $d$ of the identity permutation. Since $d_{\max }(\iota, \sigma)=d_{\max }(\pi, \pi \sigma)$, the number of permutations in $S_{n}$ within Chebyshev distance $d$ of any permutation $\pi \in S_{n}$ will also be $V(n, d)$. Bounds on $P(n, a)$ and $V(n, d)$ will be considered in Sec. 4 ,

\section{Constructions}

In this section we give a number of constructions of PAs, one explicit and some recursive.

\subsection{An explicit construction}

Let $n$ and $d$ be given. Define

$$
C=\left\{\left(\pi_{1}, \ldots, \pi_{n}\right) \in S_{n} \mid \pi_{i} \equiv i \quad(\bmod d) \text { for all } i \in[n]\right\} .
$$

If $n=a d+b$, where $0 \leq b<d$, then $C$ is an $(n, d) \mathrm{PA}$ and

$$
|C|=((a+1) !)^{b}(a !)^{d-b} .
$$

In particular, we get the following bound. 
Theorem 1. If $n=a d+b$, where $0 \leq b<d$, then

$$
P(n, d) \geq((a+1) !)^{b}(a !)^{d-b} .
$$

Example 1. For $d=2$, we get

$$
P(2 a, 2) \geq(a !)^{2} .
$$

We note that if $2 d>n$, then $a=1$ and $b=n-d$ and so $|C|=2^{n-d}$. If $2 d=n$, then $a=2, b=0$, and we have $|C|=2^{d}=2^{n-d}$ as well. However, if $2 d<n$, then $|C|>2^{n-d}$. Especially, when $d$ is small relative to $n,|C|$ is much larger than $2^{n-d}$. For example, for $n=30, d=2,|C| / 2^{n-d} \approx 6.37 \times 10^{15}$.

This construction has a very simple decoding algorithm. For $d \geq 2 t+1$, we can correct error up to size $t$ in any coordinate. For coordinate $i$, the codeword has value $\pi_{i} \equiv i(\bmod d)$. Suppose that this coordinate is changed into $\sigma=$ $\pi_{i}+u$, where $|u| \leq t$. Then $\pi_{i}$ is the integer congruent to $i$ which is closest to $\sigma$. Therefore, decoding of position $i$ is done by first computing

$$
a \equiv i-\sigma \quad(\bmod d),
$$

where $-(d-1) / 2 \leq a \leq(d-1) / 2$. Then $a=-u$, and so we decode into $\sigma+a=\pi_{i}$.

\subsection{First recursive construction}

Let $C$ be an $(n, d) \mathrm{PA}$ of size $M$, and let $r \geq 2$ be an integer. We define an $(r n, r d) \mathrm{PA}, C_{r}$, of size $M^{r}$ as follows: for each multi-set of $r$ code words from $C$,

$$
\left(\pi_{1}^{(j)}, \ldots, \pi_{n}^{(j)}\right), j=0,1, \ldots, r-1,
$$

let

$$
\rho_{j}=\left(r \pi_{1}^{(j)}-j, \ldots, r \pi_{n}^{(j)}-j\right), j=0,1, \ldots, r-1,
$$

and include $\left(\rho_{0}\left|\rho_{1}\right| \ldots \mid \rho_{r-1}\right)$ as a codeword in $C_{r}$. It is clear that under this construction the distance between any two distinct $\rho_{j}, \rho_{j^{\prime}}$ is at least $r d$. It is also easy to check that $\left(\rho_{0}\left|\rho_{1}\right| \ldots \mid \rho_{r-1}\right) \in S_{r n}$. Hence $\left|C_{r}\right|=P(n, d)^{r}$. In particular, we get the following bound.

Theorem 2. If $n>d$ and $r \geq 2$, then

$$
P(r n, r d) \geq P(n, d)^{r} .
$$

\subsection{Second recursive construction}

For a permutation $\pi=\left(\pi_{1}, \pi_{2}, \ldots, \pi_{n}\right) \in S_{n}$ and an integer $m, 1 \leq m \leq n+1$ define

$$
\varphi_{m}(\pi)=\left(m, \pi_{1}^{\prime}, \pi_{2}^{\prime}, \ldots, \pi_{n}^{\prime}\right) \in S_{n+1}
$$


by

$$
\begin{array}{ll}
\pi_{i}^{\prime}=\pi_{i} & \text { if } \pi_{i} \leq m, \\
\pi_{i}^{\prime}=\pi_{i}+1 & \text { if } \pi_{i}>m .
\end{array}
$$

Let $C$ be an $(n, d) \mathrm{PA}$, and let

$$
1 \leq s_{1}<s_{2}<\cdots<s_{t} \leq n+1
$$

be integers. Define

$$
C\left[s_{1}, s_{2}, \ldots, s_{t}\right]=\left\{\varphi_{s_{j}}(\pi) \mid 1 \leq j \leq t, \pi \in C\right\} .
$$

Theorem 3. If $C$ is an $(n, d) P A$ of size $M$ and

$$
s_{j}+d \leq s_{j+1} \text { for } 1 \leq j \leq t-1,
$$

then $C\left[s_{1}, s_{2}, \ldots, s_{t}\right]$ is an $(n+1, d) P A$ of size $t M$.

Theorem 4. If $C$ is an $(n, d) P A$ of size $M$ and $n \leq 2 d$, then $C[d]$ is an $(n+1, d+1) P A$ of size $M$.

Proof. If $j>j^{\prime}$, then

$$
d_{\max }\left(\varphi_{s_{j}}(\pi), \varphi_{s_{j^{\prime}}}(\sigma)\right) \geq s_{j}-s_{j^{\prime}} \geq d .
$$

Next, consider $j^{\prime}=j$. If $\pi, \sigma \in C, \pi \neq \sigma$, then w.l.o.g, there exist an $i$ such that $\pi_{i} \geq \sigma_{i}+d$. Hence

$$
d_{\max }\left(\varphi_{s_{j}}(\pi), \varphi_{s_{j}}(\sigma)\right) \geq \begin{cases}\pi_{i}-\sigma_{i}+1>d & \text { if } \pi_{i}>s_{j} \geq \sigma_{i} \\ \pi_{i}-\sigma_{i} \geq d & \text { otherwise }\end{cases}
$$

This proves Theorem 3. To complete the proof of Theorem 4 we note that

$$
\pi_{i} \geq \sigma_{i}+d \geq d+1>d
$$

and

$$
\sigma_{i} \leq \pi_{i}-d \leq n-d \leq d
$$

Hence $\pi_{i}>d \geq \sigma_{i}$ and so

$$
d_{\max }\left(\varphi_{s_{j}}(\pi), \varphi_{s_{j}}(\sigma)\right) \geq d+1 .
$$

The constructions imply bounds on $P(n, d)$. First, choosing $t=\lfloor n / d\rfloor+1$, $s_{t}=n+1$ and $s_{j}=(j-1)\lfloor n / d\rfloor+1$ for $1 \leq j \leq t-1$, we get the following bound.

Theorem 5. If $n>d \geq 1$, then

$$
P(n+1, d) \geq\left(\left\lfloor\frac{n}{d}\right\rfloor+1\right) P(n, d) .
$$


Example 2. In Example 1 we showed that the explicit construction implied that $P(2 a, 2) \geq(a !)^{2}$. Combining Theorem 5 and search, we can improve this bound. We have found that $P(7,2) \geq 582$, see the table at the end of the next section. From repeated use of Theorem 5 we get

$$
P(2 a, 2) \geq(a(a-1) \cdots 5)^{2} \cdot 4 P(7,2) \geq \frac{97}{24}(a !)^{2} .
$$

Theorem 4 implies the following bound.

Theorem 6. If $d<n \leq 2 d$, then

$$
P(n+1, d+1) \geq P(n, d) .
$$

Theorem [6 shows in particular that for a fixed $r$,

$$
P(d+1+r, d+1) \geq P(d+r, d) \text { for } d \geq r .
$$

We will show that $P(d+r, d)$ is bounded. We show the following theorem.

Theorem 7. For fixed $r$, there exist constants $c_{r}$ and $d_{r}$ such that $P(d+r, d)=$ $c_{r}$ for $d \geq d_{r}$. Moreover,

$$
c_{r} \leq 2^{2 r}(2 r) !
$$

and

$$
d_{r} \leq 1+(2 r-1) c_{r}-r .
$$

Remark. The main point of Theorem $\square$ is the existence of $c_{r}$ and $d_{r}$. The actual bounds given are probably quite weak in general. For example, Theorem 7 gives the bounds $c_{1} \leq 8$ and $d_{1} \leq 8$. In Theorem 8 below, we will show that $c_{1}=3$ and $d_{1}=2$. Theorem 7 gives $c_{2} \leq 384$ and $d_{2} \leq 1151$, whereas numerical computation indicate that $c_{2}=9$ and $d_{2}=5$.

We split the proof of Theorem 7 into three lemma.

Lemma 1. If $d \geq r$, then $P(d+r, d) \leq 2^{2 r}(2 r)$ !.

Proof. Suppose that there exists an $(d+r, d)$ PA $C$ of size $M>2^{2 r}(2 r) !$. We call the integers

$$
1,2, \ldots, r \text { and } d+1, d+2, \ldots, d+r
$$

potent, the first $r$ smaller potent, the last $r$ larger potent. Two potent integers are called equipotent if both are smaller potent or both are larger potent. If the distance between two permutations $\left(\pi_{1}, \pi_{2}, \ldots, \pi_{n}\right),\left(\rho_{1}, \rho_{2}, \ldots, \rho_{n}\right)$ is at least $d$, then there exists some position $i$ such that, w.l.o.g, $\pi_{i}-\rho_{i} \geq d$, Then $\pi_{i}$ is a larger potent element and $\rho_{i}$ is smaller potent. Each permutation in $S_{d+r}$ contains $2 r$ potent elements and we call the set of positions of these the potency support $\chi(\pi)$ of the permutation, that is, the potency support of $\pi$ is

$$
\chi(\pi)=\left\{i \mid 1 \leq \pi_{i} \leq r\right\} \cup\left\{i \mid d+1 \leq \pi_{i} \leq d+r\right\} .
$$


The potency support of $C$ is the union of the potency support of the permutations in $C$, that is

$$
\begin{aligned}
\chi(C)= & \left\{i \mid 1 \leq \pi_{i} \leq r \text { for some } \pi \in C\right\} \\
& \cup\left\{i \mid d+1 \leq \pi_{i} \leq d+r \text { for some } \pi \in C\right\} .
\end{aligned}
$$

Let $\pi \in C$. For each $\rho \in C, \rho \neq \pi$, we have $d(\pi, \rho) \geq d$. Hence there exists some $i \in \chi(\pi)$ such that $\rho_{i}$ is potent. Therefore, the set

$$
\{(\rho, i) \mid \rho \in C \text { and } i \in \chi(\pi)\}
$$

contains at least $2 r+(M-1)>M$ elements. Hence there is an $i \in \chi(\pi)$ such that

$$
\mid\left\{\rho \in C \mid \rho_{i} \text { is potent }\right\} \mid>M /(2 r)>2^{2 r}(2 r-1) !
$$

Since

$$
\begin{aligned}
\left\{\rho \in C \mid \rho_{i} \text { is potent }\right\}= & \left\{\rho \in C \mid \rho_{i} \text { is smaller potent }\right\} \\
& \cup\left\{\rho \in C \mid \rho_{i} \text { is larger potent }\right\},
\end{aligned}
$$

there exists a subset $C_{1} \subset C$ such that

$$
\left|C_{1}\right|>2^{2 r-1}(2 r-1) \text { ! }
$$

and the elements in position $i_{1}=i$ are equipotent.

We can now repeat the procedure. Let $\pi \in C_{1}$. There must exist an $i_{2} \in$ $\chi(\pi) \backslash\left\{i_{1}\right\}$ such that

$$
\mid\left\{\rho \in C_{1} \mid \rho_{i_{2}} \text { is potent }\right\}|\geq| C_{1} \mid /(2 r-1)>2^{2 r-1}(2 r-2) ! .
$$

Hence we get subset $C_{2} \subset C_{1}$ such that

$$
\left|C_{2}\right|>2^{2 r-2}(2 r-2) \text { ! }
$$

and the elements in position $i_{2}$ are equipotent (and the elements in position $i_{1}$ are equipotent).

Repeated use of the same argument will produce for each $j, 1 \leq j \leq 2 r$ a set $C_{j}$ such that

$$
\left|C_{j}\right|>2^{2 r-j}(2 r-j) !
$$

and for $j$ positions $i_{1}, i_{2}, \ldots i_{j}$, the elements in those positions are all equipotent. In particular, $\left|C_{2 r}\right|>1$, all permutations in $C_{2 r}$ have the same potency support $\left\{i_{1}, i_{2}, \ldots, i_{2 r}\right\}$, and for each of these positions, all the elements in that position are equipotent. This is a contradiction since the distance between two such permutations must be less than $d$. Hence the assumption that a PA of size larger than $2^{2 r}(2 r)$ ! exists leads to a contradiction.

Lemma 1 combined with (1) proves the existence of $c_{r}$ and $d_{r}$ and gives the bound (2). 
Lemma 2. If $C$ is $a(d+r, d) P A$ of size $M$ where

$$
d>r \text { and } d+r>|\chi(C)|,
$$

then there exists a $(d-1+r, d-1) P A$ of size $M$. In particular, if $M=P(d+r, d)$, then

$$
P(d-1+r, d-1)=P(d+r, d) .
$$

Proof. Replace all elements in range $r+1, r+2, \ldots, d$ in the permutations of $C$ by a star * which will denote "unspecified". The permutations in $C$ is transformed into vectors containing the potent elements and $d-r$ stars. Note that if we replace the unspecified elements in each vector by the integers $r+$ $1, r+2, \ldots, d$ in some order, we get a permutation, and the distance between two such permutations will be at least $d$ since we have not changed the potent elements.

Since the length $d+r$ of $C$ is larger than $|\chi(C)|$, there exists a position where all the vectors contains a star. Remove this position from each vector and reduce all the larger potent elements by one. This given a set of $M$ vectors of length $d-1+r$ and such that the distance between any two is at least $d-1$. Replacing the $d-1-r$ stars in each vector by $r+1, r+1, \ldots, d-1$ in some order, we get an $(d-1+r, d-1) \mathrm{PA}$ of size $M$.

If $M=P(d+r, d)$, then we get

$$
P(d-1+r, d-1) \geq P(d+r, d) .
$$

Since $P(d-1+r, d-1) \leq P(d+r, d)$ by (1) , the lemma follows.

Lemma 3. If $C$ is a $(d+r, d) P A$ of size $M$ and $d \geq r$, then

$$
|\chi(C)| \leq M(2 r-1)+1
$$

Proof. Each permutation has potency support of size $2 r$. The potency support of any two permutations in $C$ must overlap since their distance is at least $d$. Hence each permutation after the first will contribute at most $2 r-1$ new elements to the total potency support. Therefore,

$$
|\chi(C)| \leq 2 r+(M-1)(2 r-1) .
$$

Remark. By a more involved analysis, we can improve this bound somewhat. For example, we see that two new permutations can contribute at most $4 r-3$ to the total support.

We can now complete the proof of Theorem 7 Let $C$ be a $(d+r, r)$ code of size $c_{r}$. By Lemma 3, $|\chi(C)| \leq c_{r}(2 r-1)+1$. If $d>1+c_{r}(2 r-1)-r$, then $d+r>|\chi(C)|$. Hence, by Lemma 2, $P(d-1+r, d-1)=P(d+r, d)$. Therefore, $d_{r} \leq 1+c_{r}(2 r-1)-r$, that is, (3) is satisfied. This completes the proof of Theorem 7 . 
Theorem 8. We have $P(d+1, d)=3$ for $d \geq 2$.

Proof. We use the same notation as in the proof of Lemma 2. Let $C$ be an $(d+1, d)$ PA. The only potent elements are 1 and $n$. W.lo.g. we may assume the first permutation in $C$ is $(1, n, *, *, \ldots)$ where $*$ denotes some unspecified integer in the range $2,3, \ldots, d$. W.l.o.g, a second permutation has one of three forms:

$$
(n, 1, *, *, \ldots),(n, *, 1, *, \ldots),(*, 1, n, *, \ldots) .
$$

We see that if the second permutation is of the first form, there cannot be more permutations. If the second permutation is of the form $(n, *, 1, *, \ldots)$, then there is only one possible form for a third permutation, namely $(1, *, n, *, \ldots)$. Hence we see that $P(d+1, d) \leq 3$ and that $P(d+1, d)=3$ for $d \geq 2$.

To determine $P(d+r, d)$ along the same lines for $r \geq 2$ seems to be difficult because of the many cases that have to be considered. Even to determine $P(d+2, d)$ will involve a large number of cases. For example for the second permutation there are 138 essentially different possibilities for the four positions in the potency support of the first permutation. For each of these there are many possible third permutations, etc.

\subsection{Encoding/decoding of some PA constructed by the second recursive construction}

Suppose we start with the PA

$$
C_{d}=\{(1,2,3, \ldots, d)\} .
$$

For $\nu=d, d+1, \ldots, n-1$ let

$$
C_{\nu+1}=C_{\nu}[1, \nu+1] .
$$

Then $C_{n}$ is an $(n, d) \mathrm{PA}$ of size $2^{n-d}$. For some applications, we may want to map a set of binary vectors to a permutation array. One algorithm for mapping a binary vector $\left(x_{1}, x_{2}, \ldots, x_{n-d}\right)$ into $C_{n}$ would be to use the recursive construction of $C_{n}$ by mapping $\left(x_{1}, x_{2}, \ldots, x_{i}\right)$ into a permutation $\pi$ in $C_{d+i}$. Recursively, we can then map $\left(x_{1}, x_{2}, \ldots, x_{i}, 0\right)$ to $f_{1}(\pi)$ and $\left(x_{1}, x_{2}, \ldots, x_{i}, 1\right)$ to $f_{d+i+1}(\pi)$.

However, there is an alternative algorithm which requires less work. Retracing the steps of the construction, we see that given some initial part of length less than $n-d$ of a permutation in $C_{n}$, there are exactly two possibilities for the next element, one "larger" and one "smaller". More precisely, induction shows that if the initial part of length $i-1$ contains exactly $t$ "smaller" elements, then element number $i$ is either $t+1$ (the "smaller") or $n-i+t+1$ (the "larger"). This is the basis for a simple mapping from $Z_{2}^{n-d}$ to $C_{n}$. We give this algorithm in Figure 1.

We see that the difference between the larger and the smaller element in position $i \leq n-d$ is $n-i$. Hence we can recover from any error of size less than 


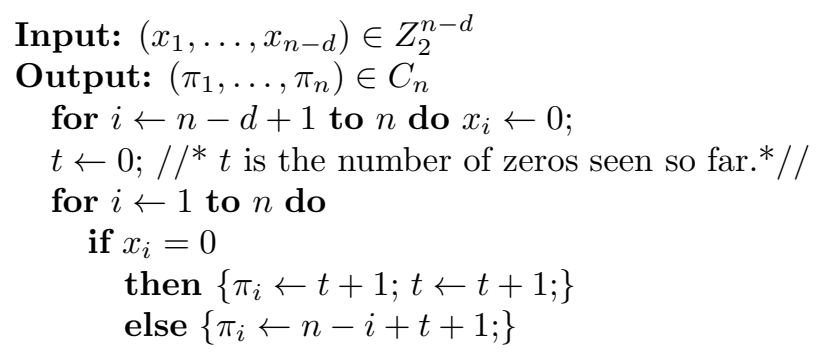

Figure 1: Algorithm mapping $Z_{2}^{n-d}$ to $C_{n}$

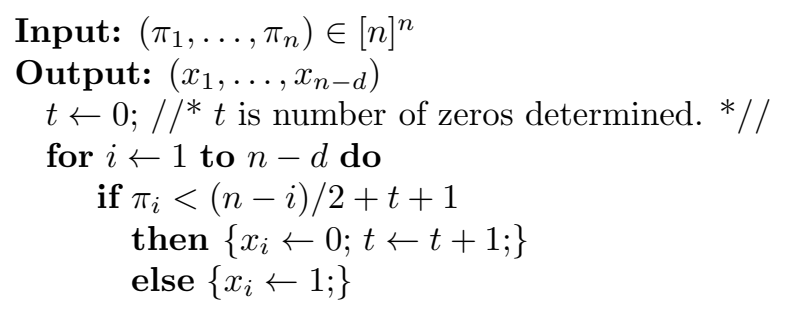

Figure 2: Decoding algorithm recovering the binary preimage from a corrupted permutation in $C_{n}$. 
$(n-i) / 2$ by choosing the closest of the two possible values, and the corresponding binary value. We give the decoding algorithm in Figure 2.

Without going into all details, we see that we can get a similar mapping from $q$-ary vectors. Now we start with the PA

$$
C_{(q-1) d}=\{(1,2,3, \ldots,(q-1) d)\} .
$$

For $(q-1) d \leq \nu \leq n-1$ let $s_{j}=(j-1)\lfloor\nu /(q-1)\rfloor+1$ for $1 \leq j \leq q-1$ and $s_{q}=\nu+1$. Let

$$
C_{\nu+1}=C_{\nu}\left[s_{1}, s_{2}, \ldots, s_{q}\right] \text {. }
$$

Then $C_{n}$ is an $(n, d)$ PA of size $q^{n-(q-1) d}$. Encoding and decoding correcting errors of size at most $(d-1) / 2$, based on the recursion, is again relatively simple.

\section{Further bounds on $P(n, d)$}

\subsection{General bounds}

Since $d_{\max }(\pi, \sigma) \leq n-1$ for any two distinct permutations in $S_{n}$, we have $P(n, n)=1$. Therefore, we only consider $d<n$.

Since the spheres of radius $d$ in $S_{n}$ all have size $V(n, d)$, we can get a Gilbert type lower bound on $P(n, d)$.

Theorem 9. For $n>d \geq 2$ we have

$$
P(n, d) \geq \frac{n !}{V(n, d-1)} .
$$

Proof. It is clear that the following greedy algorithm produces a permutation array with cardinality at least $n ! / V(n, d-1)$.

1. Start with any permutation in $S_{n}$.

2. Choose a permutation whose distance is at least $d$ to all previous chosen permutations.

3. Repeat step 2 as long as such a permutation exists.

Let $C$ be the permutation array produced by the above greedy algorithm. Once the algorithm stops, $S_{n}$ will be covered by the $|C|$ spheres of radius $d-1$ centered at the code words in $C$. Thus $n ! \leq|P| \cdot V(n, d-1)$ which implies our claim.

Similarly, since the spheres $V(n,\lfloor(d-1) / 2\rfloor)$ are disjoint, we get the following Hamming type upper bound.

Theorem 10. If $n>d \geq 1$, then

$$
P(n, d) \leq \frac{n !}{V(n,\lfloor(d-1) / 2\rfloor)} .
$$


If $n \leq 2 d$ and $d$ is even, we can combine the bound in Theorem 10 with Theorem [6 to get the following bound which is stronger than the ordinary Hamming bound, at least in the cases we have tested.

Theorem 11. If $d$ is even and $2 d \geq n>d \geq 2$, then

$$
P(n, d) \leq \frac{(n+1) !}{V(n+1, d / 2)} .
$$

Example 3. For $n=11$ and $d=6$, Theorem 10 gives

$$
P(11,6) \leq\left\lfloor\frac{11 !}{V(11,2)}\right\rfloor=\left\lfloor\frac{11 !}{11854}\right\rfloor=3367
$$

whereas Theorem 11 gives

$$
P(11,6) \leq\left\lfloor\frac{12 !}{V(12,3)}\right\rfloor=\left\lfloor\frac{12 !}{563172}\right\rfloor=850 .
$$

Remark. We can of course use Theorem 6 repeatedly $r$ times and then Theorem 10 to get

$$
P(n, d) \leq \frac{(n+r) !}{V(n+r,\lfloor(d+r-1) / 2\rfloor)}
$$

for all $r \geq 0$. However, it appears we get the best bounds for $r=1$ when $d$ is even and $r=0$ when $d$ is odd.

In general, no simple expression of $V(n, d)$ is known. A survey of known results as well as a number of new results on $V(n, d)$ were given by Kløve [8]. Here we briefly give some main results.

As observed by Lehmer [9, $V(n, d)$ can be expressed as a permanent. The permanent of an $n \times n$ matrix $A$ is defined by

$$
\operatorname{per} A=\sum_{\pi \in S_{n}} a_{1, \pi_{1}} \cdots a_{n, \pi_{n}} .
$$

In particular, if $A$ is a $(0,1)$-matrix, then

$$
\operatorname{per} A=\mid\left\{\pi \in S_{n}: a_{i, \pi_{i}}=1 \text { for all } i\right\} \mid .
$$

Let $A^{(n, d)}$ be the $n \times n$ matrix with $a_{i, j}^{(n, d)}=1$ if $|i-j| \leq d$ and $a_{i, j}^{(n, d)}=0$ otherwise.

Lemma 4. $V(n, d)=\operatorname{per} A^{(n, d)}$.

Proof.

$$
\begin{aligned}
V(n, d) & =\left|\left\{\pi \in S_{n}: d_{\max }(\iota, \pi) \leq d\right\}\right| \\
& =\mid\left\{\pi \in S_{n}:\left|i-\pi_{i}\right| \leq d \text { for all } i\right\} \mid \\
& =\mid\left\{\pi \in S_{n}: a_{i, \pi_{i}}^{(n, d)}=1 \text { for all } i\right\} \mid \\
& =\operatorname{per} A^{(n, d)} .
\end{aligned}
$$


For fixed $d, V(n, d)$ satisfies a linear recurrence in $n$. A proof is given in [10] (Proposition 4.7.8 on page 246). For $1 \leq d \leq 3$ these recurrences were determined explicitly by Lehmer [9], and for $4 \leq d \leq 6$ by Kløve [8]. In particular, this implies that

$$
\lim _{n \rightarrow \infty} V(n, d)^{1 / n}=\mu_{d},
$$

where $\mu_{d}$ is the largest root of the minimal polynomial corresponding to the linear recurrence of $V(n, d)$. Lehmer [9] determined $\mu_{d}$ approximately for $d=$ $1,2,3$ and Kløve 8 for $d \leq 8$.

For an $n \times n(0,1)$-matrix it is known (see Theorem 11.5 in [11]) that

$$
\operatorname{per} A \leq \prod_{i=1}^{n}\left(r_{i} !\right)^{1 / r_{i}},
$$

where $r_{i}$ is the number of ones in row $i$.

For $A^{(n, d)}$ we clearly have $r_{i} \leq 2 d+1$ for all $i$. Hence

$$
V(n, d) \leq[(2 d+1) !]^{n /(2 d+1)} \text { for all } n
$$

and

$$
\mu_{d} \leq[(2 d+1) !]^{1 /(2 d+1)} .
$$

In Table 1 we give $\mu_{d}$ and this upper bound.

Table 1: $\mu_{d}$ and its upper bound.

\begin{tabular}{|c|c|c|c|}
\hline$d$ & $\mu_{d}$ & {$[(2 d+1) !]^{1 /(2 d+1)}$} & $\mu_{d} /(2 d+1)$ \\
\hline 1 & 1.61803 & 1.81712 & 0.53934 \\
2 & 2.33355 & 2.60517 & 0.46671 \\
3 & 3.06177 & 3.38002 & 0.43739 \\
4 & 3.79352 & 4.14717 & 0.42150 \\
5 & 4.52677 & 4.90924 & 0.41152 \\
6 & 5.26082 & 5.66769 & 0.40468 \\
7 & 5.99534 & 6.42342 & 0.39969 \\
8 & 6.73016 & 7.17704 & 0.39589 \\
\hline
\end{tabular}

We note that for large $d, \mu_{d} /(2 d+1) \approx 1 / e$.

Combining Theorem 9 and (4) we get

Corollary 1. For $n>d \geq 1$, we have

$$
P(n, d) \geq \frac{n !}{[(2 d-1) !]^{n /(2 d-1)}} .
$$




\subsection{Table of bounds on $P(n, d)$}

We have used the following greedy algorithm to find an $(n, d)$ PA $C$ : Let the identity permutation in $S_{n}$ be the first permutation in $C$. For any set of permutations chosen, choose as the next permutation in $C$ the lexicographically next permutation in $S_{n}$ with distance at least $d$ to the chosen permutations in $C$ if such a permutation exists. The size of the resulting PA is of course a lower bound on $P(n, d)$.

The lower bounds in Table 2 were in most cases found by this greedy algorithm. For $n=8, d=5$, the greedy algorithm gave a PA of size 26. However,

$$
P(8,5) \geq P(7,4) \geq 28
$$

by Theorem 6. Similarly,

$$
P(10,7) \geq P(9,6) \geq P(8,5) \geq 28 .
$$

Some other of the lower bounds are also determined using Theorem 6. They are marked by $*$. The upper bound is the Hamming type bound in Theorem 10 or it's modified bound in Theorem [11. Since $P(n, 1)=n$ ! for all $n$, this is not included in the table.

\begin{tabular}{|c|c|c|c|}
\hline & $d=2$ & $d=3$ & $d=4$ \\
\hline$n=d+1$ & 3 & 3 & 3 \\
\hline$n=d+2$ & $6-24$ & 9 & $9-12$ \\
\hline$n=d+3$ & $29-120$ & $20-34$ & $28-43$ \\
\hline$n=d+4$ & $90-720$ & $84-148$ & $68-166$ \\
\hline \multirow[t]{2}{*}{$n=d+5$} & $582-5040$ & $401-733$ & $283-4077$ \\
\hline & $d=5$ & $d=6$ & $d=7$ \\
\hline$n=d+1$ & 3 & 3 & 3 \\
\hline$n=d+2$ & $9-12$ & $9-18$ & $9-18$ \\
\hline$n=d+3$ & $28^{*}-43$ & $28^{*}-60$ & $28^{*}-60$ \\
\hline$n=d+4$ & $95-166$ & $95^{*}-216$ & $95^{*}-216$ \\
\hline$n=d+5$ & $236-714$ & $236^{*}-850$ & $236^{*}-850$ \\
\hline
\end{tabular}

Table 2: Bounds on $P(n, d)$.

\section{Conclusion}

We give a number of constructions of permutations arrays under the Chebyshev distance, some with efficient decoding algorithms. We also consider an explicit mapping of vectors to permutations with efficient encoding/decoding. Finally, we give some bounds on the size of PAs under the Chebyshev distance. 


\section{References}

[1] A. Jiang, R. Mateescu, M. Schwartz and J. Bruck, "Rank Modulation for Flash Memories," in Proc. IEEE Internat. Symp. on Inform. Th., 2008, pp. 1731-1735.

[2] A. Jiang, M. Schwartz and J. Bruck, "Error-Correcting Codes for Rank Modulation," in Proc. IEEE Internat. Symp. on Inform. Th., 2008, pp. 1736-1740.

[3] P. Diaconis, Group Representations in Probability and Statistics. Hayward, CA: Institute of Mathematical Statistics, 1988.

[4] M. Kendall and J. D. Gibbons, Rank correlation methods. London, U.K.: Edward Arnold, 1990.

[5] E. Stoll and L. Kurz, "Suboptimum Rank Detection Procedures Using Rank Vector Codes," IEEE Trans. Commun., vol. COM-16, pp. 402- 410, June 1968.

[6] H. Chadwick, L. Kurz, "Rank permutation group codes based on Kendall's correlation statistic," IEEE Trans. Inform. Th., vol. IT-15, pp. 306-315, Mar 1969.

[7] S. Haykin, Communication Systems, 4th Ed. John Wiley \& Sons, 2001.

[8] T. Kløve, "Spheres of Permutations under the Infinity Norm - Permutations with limited displacement," Reports in Informatics, Dept. of Informatics, Univ. Bergen, Report no. 376, November 2008.

[9] D. H. Lehmer, "Permutations with strongly restricted displacements," in Combinatorial Theory and its Applications II, P. Erdös, A. Rńyi and V. T. Sós (eds.), Amsterdam: North Holland Publ., 1970.

[10] R. P. Stanley, Enumerative Combinatorics, Vol. I. Cambridge, U.K.: Cambridge Univ. Press, 1997.

[11] J.H. van Lint, R. M. Wilson, A Course in Combinatorics., 2nd ed. Cambridge, U.K.: Cambridge Univ. Press, 2001. 